Perhaps this ethos has always been so much beyond reproach that no improvement was needed. But I suspect that it was rather because it was taken for granted and, indeed, overlooked. Twenty years ago I remember several examiners remarking to me, "That man was unkind to his patient. I don't think he's fit to be a doctor. I shall fail him." Recently, I have not heard this. Again, I suspect this is because of the rule of the pedants: "You can't," they will say, "fail a man in his examination because he is unkind to his patients. That isn't what examinations are for. He knows his work." And so once more it is the attitude of the potential doctor to his patient and to his work that goes to the wall. It doesn't matter.

Here let me say that one of the major endemic diseases in our society is that disorder that thirsts for examinations. Examinations are a rash or morbid eruption on the body of democracy. They are thought to be fair. The current thirst is to make them even fairer by making them so objective that they can be marked by a computer-the device known in America as the multiple-choice method. However, what is overlooked is that, like other tricks, this trick can be learned, and a high performance in such tests is not synonymous with profound knowledge. What the enthusiasts for such methods overlook entirely is the educational function of examinations. If it becomes known that a sympathetic, understanding, and kindly attitude is also valued, and indeed required, then that attitude is the more likely to be forthcoming and indeed become a habit.

\section{Conclusion}

I have already outlined why the State monopoly of medical practice provides conditions that are not unfavourable to the " 9 to 5 " mentality, and the substitution of the form for the substance. If this happens the chief victims will be the public, their patients. But medicine, too, will have lost something, and I for one would not really care to be connected with it any more. The National Health Service has so much good in it that there would be few amongst us who would care to bring back the old days. All the more reason for protecting it from this its greatest danger by trying to ensure that those we take into medicine go into it because they feel some vocational call, and have the right kind of character as well as intellect.

Thus I come back to my text. The welfare state is one of the most substantial attempts to translate an ideal into practice. Naturally, not all of its implications were foreseen. Medicine perhaps presents the outstanding example of how greatly the realization of this modern attempt at Utopia depends on character, on an attitude of mind. I do not think this has yet been realized. And I fear that, unless it is, our attempt at Utopia will destroy itself. Manners makyth Man.

\title{
BILIARY AND RENAL COLIC
}

\author{
BY \\ E. B. FRENCH, M.B., F.R.C.P., F.R.C.P.Ed. \\ W. A. T. ROBB, M.B., F.R.C.S.; F.R.C.S.Ed. \\ Western General Hospital, Edinburgh
}

It is popularly believed that all pain described as colic is paroxysmal or griping in character. Whereas this is often true when pain arises in the small or large intestine (whence the word is derived, " colike ... ingendreth in a gutte named colon "-Shorter O.E.D.), only rarely is it true of the pain of biliary or renal colic. In these instances, therefore, the term is misleading and may be responsible for a common misconception of the type of pain usually associated with biliary and ureteric calculus.

The belief that the pain of these colics has an intermittent wave-like pattern is evidently fairly widespread, for it has frequently been encountered in discussion with colleagues and during the teaching of students and postgraduates in London, Edinburgh, and Glasgow. Other people take the view that a colic is any pain which makes the patient restless. Though this is commonly true of patients with biliary and renal colic, some prefer to lie still, while a few with pain from acute myocardial infarction may pace about for relief.

It is obvious that familiarity with the usual features of a pain and its commoner variants is of immense diagnostic value. The present inquiry was made to emphasize in particular the time/intensity patterns of biliary and renal colic, but it was finally prompted by the unusual experience of meeting a patient with cholelithiasis in which the pain was truly intermittent. One of us (E. B. F.) studied and analysed the symptoms of 50 consecutive cases with a final diagnosis of gall-stone colic which were admitted to the gastro-intestinal unit at the Western General Hospital, Edinburgh. Similarly W. A. T. R. studied 50 consecutive patients admitted with "renal" colic to the urological unit.
Methods
Choice of Patients.-When a patient was admitted to these units with a provisional diagnosis of biliary or renal colic one of us was notified. Cases had been collected by transfer from other units, from out-patient consultations, or by direct admission from general practitioners.

Clinical History.-History was not obtained on a prescribed form. Particular attention was, however, paid to the analysis of pain in accordance with the scheme described by Ryle (1948). Those patients with biliary colic were also asked directly whether they had observed changes in the colour of the urine or stool, and also whether they had had previous indigestion. In renal colic direct inquiry was made about difficulty or discomfort on urination or about discoloration of the urine.

Validity of History.-In biliary colic this was assessed in three ways. (1) The description of pain was compared with that recorded in the resident's notes. (2) A senior student was asked to question 10 consecutive patients of the series about their symptoms in accordance with the method of analysis of pain described by Ryle (1948). It was explained to the student that the object of this request was to find out whether pain analysis by this method would result in agreement between the two observers. (3) Parous women were asked for an account of pain in labour, as a test of the reliability of their memories. None of these tests were applied to the histories of renal colic.

\section{Biliary Colic}

Firstly a case history of a common type of attack is given in order to provide a standard with which the variations may be compared. 


\section{Case Report}

A married woman aged 49 was sitting at her work at 2.30 p.m. when she was suddenly seized with severe epigastric pain, which became progressively worse over the next halfhour until it was excruciating. It spread to the right hypochondrium and through to the back below the right shoulderblade. She managed to make her way home, which was near by. Soon she applied a hot-water bottle to her side and sat by the fire, but the pain continued, so she tried walking about and took some baking-soda. She was restless and perspiring and later she vomited several times, but without relief. Her husband arrived home just after 5 p.m. and at once remarked that she was pale. He gave her some whisky and sent for the doctor, who arrived about an hour later. She was given an injection of morphine, though the pain had already begun to settle, and was admitted to hospital. She slept soundly and awoke next morning feeling well.

Three months previously she had had a similar attack, attributed by her to indigestion. Her appetite was normal and she was not overweight. There was no family history of gallstones.

On examination the following day her conjunctivae were yellow and bile pigment was found in the urine. Tenderness was present in the right hypochondrium. Straight $x$-ray examination showed many radio-opaque stones in the gallbladder and one presumed to be in the common bile-duct.

When asked to compare the pain with her experience of childbirth she stated that labour pains were not so bad, as they came and went, whereas her recent pain was comparable to the last pains of labour, was severe at onset, though it steadily became worse, and once at its peak it was constant for over two hours until it began to diminish gradually just before the injection.

\section{Results}

Time/Intensity Pattern of Pain.-Few patients attempted to assign any quality to the pain, as they were preoccupied by its severity. Some requested operation after a single attack as they feared the possibility of a recurrence. In 15 cases the maximum severity of the pain was present at its onset, similar to that of a perforated peptic ulcer. In 16 cases the intensity rose to its maximal severity in 10 minutes to one hour, whereas in the rest the pain only reached its maximum after several hours. The mode of cessation of the pain was often obscured by treatment; without treatment it ceased gradually in most cases, but abruptly in seven patients, in six of whom the onset was

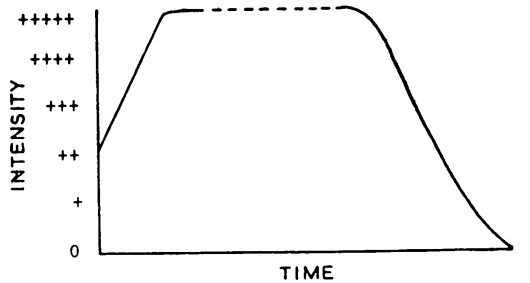

FIG. 1.-Biliary colic: 30 cases; renal colic: 17 cases.

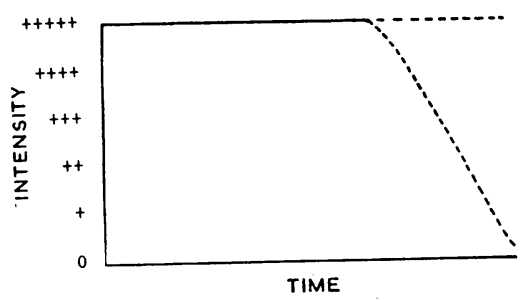

FIg. 3.-Maximum intensity at onset; sudden or gradual cessation. Biliary colic, 15 cases; renal colic, 12 cases. also abrupt. The pain when at its maximal severity was continuous, without any waxing or waning, in 45 cases. Three patients observed that excruciating exacerbations lasting 10 to 15 minutes were superimposed on a background of very severe pain. One woman described a dull constant pain accentuated by the pulse-beating. One woman described increasingly severe spasms each lasting several seconds, with intervals of complete freedom varying from a few seconds up to a minute. The main patterns of pain are illustrated diagrammatically in Figs. 1-4.

Frequency and Duration of Attacks.-Six patients had had no previous attacks; 30 had suffered previously on one to five occasions; and the rest had had numerous attacks. Rarely two attacks occurred in one day, and usually the pain-free interval was weeks, months, or years -in one case as long as 10 years. Treatment shortened the natural course of the pain in many instances. Half of the attacks for which patients were admitted lasted for 15 minutes to two hours, but half continued for many hours or even a day or two.

Site and Radiation of Pain.-The main sites indicated as the origin of the pain were the epigastrium (34 cases), right hypochondrium (10 cases), while the left hypochondrium, the midline of the back, below the left scapula, deep to the sternum, the hypogastrium, and to the right of the umbilicus were mentioned in one case each. Some patients noted that the first attack or the earliest attacks originated in a different site from later ones. There was no radiation in 11 cases (10 epigastric ; 1 in the right hypochondrium). Other patients indicated radiation to one or more sites as follows: below right scapula in 18, right hypochondrium in 12 , epigastrium in 7 , dorso-lumbar region of back in 6 , and shoulder-tip (right) in 5 . Pain is felt rarely in other sites: of special interest is the history of a woman who had several attacks over a period of four years; the pain began in the epigastrium and radiated to the right hypochondrium, through to the back, and also to the right breast, shoulder, arm, and forearm as far as the wrist.

Associated Features.-During the attacks patients were active and restless in 45 cases, but five preferred to sit or lie still. Sweating was mentioned by 35 patients and vomiting by 38 , in one of whom at least this might have been due to morphine as it occurred only after an injection. After the attack 11 patients complained of soreness in the right hypochondrium, two mentioned nausea, one tiredness, whereas 39 claimed that they felt quite well as soon as an attack was over.

Indigestion.-Of the 50 patients, two were known to have a peptic ulcer and one a hiatus hernia. Two suffered from heartburn, 10 complained of flatulence, of being upset by greasy food, or of occasional epigastric pain. Thirty-five stated that they had had no indigestion prior to their first attack of biliary colic.

Validity of History-taking.-A considerable error of observation may be present in any clinical analysis, especially if an attempt is being made to prove a preconceived idea. In this present series the frequency of different pain patterns was being sought with possible bias conditioned by the observer's previous belief. In renal colic bias was towards intermittent pain, whereas in biliary colic it favoured continuous pain.
FIG. 4.-Intermittent pain-true " colic." One case of biliary colic; no case of renal colic. 
In biliary colic the time/intensity pattern of pain was checked as far as possible in the three ways already mentioned. (1) In 30 cases there was agreement with the description given in the resident's notes; in 19 the description was not clear enough to decide what was meant; and in one case there was disagreement. The notes stated that the pain was continuously severe with waves of accentuation superimposed every 10 seconds, while E. B. F. obtained a history of constant severe pain accentuated by breathing. (2) There was agreement with all 10 analyses recorded by a senior student. (3) Thirty parous women gave a clear account of intermittent pain and then usually contrasted it with the continuous nature of biliary colic. Two stated that the event was so long ago that they had forgotten.

Proof of Diagnosis.-Twenty-three patients had developed jaundice after at least one of their attacks. Two who had jaundice and two others with radiological evidence of gall-stones did not undergo operation. Forty-six patients had an operation; of these, 41 had no further pains during the next five to six years, while five suffered from recurrences. Four recurrences were accompanied by jaundice: two due to stones in the common bile-duct, one due to chronic choledochitis with gross inflammatory thickening of the duct wall, and one due to a fibrous stricture at the lower end of the common duct. It is of interest that the symptoms in these attacks were identical with the original complaints in spite of the absence of the gall-bladder in each case.

\section{Renal Colic}

The following case history of a common type of attack provides a standard with which the variations may be compared.

\section{Case Report}

A previously fit man aged 27 felt a nagging pain in the left loin as he lay in bed late on a Sunday morning. The pain gradually became worse and soon after noon it was so unbearable that he was rolling about the bed. The pain gradually subsided after two to three hours from the onset. Next day the pain returned very severely and radiated round to the left side of the abdomen in front. It came and went in bouts lasting about an hour, with intervals of freedom lasting three to four hours during which he slept. During the bouts the pain was continuous, taking a few minutes to reach a peak of intensity, at which it remained until it subsided about an hour later. While urinating he felt pain at the tip of the penis and noticed that his urine was red. He was admitted to hospital on the third day, after $100 \mathrm{mg}$. of pethidine had been given by injection. Examination showed a moderate number of red cells in the urine, but no other abnormality. A small calculus was passed in the urine two days later and he had no further symptoms.

\section{Results}

Time/Intensity Pattern of Pain.-Twenty-seven of the patients assigned no quality to the pain. The remainder described a very severe ache, crushing, or stabbing qualities in about equal numbers. All agreed upon the severity of the pain, and all but two were active and restless during the attack. In half the cases the attacks started while the patients were in bed or sitting peacefully. One started while the patient was travelling in a bus. The pain was at its peak at the onset in 12 cases; in 15 the peak was reached in 15 seconds to 2 minutes, while in 23 patients the maximum intensity of the pain was not felt until 10 to 60 minutes had elapsed. In 29 instances the pain was of steady intensity. In the remainder the pain was continuous and often severe, and superimposed upon this background there were waves of greater intensity, each of these accentuations usually lasting for 10 to 30 minutes. In two cases these waves of more intense pain occurred every two to three minutes.

Frequency and Duration of Attacks.-In 30 cases there had been previous attacks. Two-thirds of the attacks lasted from a half to four hours, which is a little longer than the common range for biliary colic, though, like the latter, some pains continued for 12 or 24 hours. The natural duration was sometimes cut short by treatment.

Site and Radiation of Pain.-In seven cases the pain was felt in the renal angle only. In most others the pain in the renal angle radiated to the hypochondrium (15 cases), hypochondrium and groin or iliac fossa (14 cases), and a few to the testis, umbilicus, or the thigh. It is of particular interest perhaps that three patients claimed that the pain was felt in the hypochondrium only, and the diagnosis was put beyond all reasonable doubt in two of these by the cessation of attacks after the passage of calculi in the urine. In one patient pain in the renal angle later became more intense and radiated to the groin and testis prior to the passing of a stone three hours later. Apart from this case it was usual for a patient to feel pain in the same site even when the stone had shifted to a new site down the ureter.

Associated Features.-Nausea or vomiting and sweating were noted in about a third of these cases. Macroscopic haematuria was reported by 12 patients. Frequency was mentioned by 31 patients; in eight of them a stone was noted in the urine soon after the attack, and in three others a stone was passed a few days later. In 12 patients with frequency, calculi were demonstrated in various parts of the ureter, not at the lower end in most cases, as might have been expected from this symptom.

\section{Age and Sex Incidence}

In biliary colic, 26 of the 43 females were under 55, whereas only 1 of the 7 males was under this age. This age and sex distribution is already well known. The incidence of stones based on post-mortem studies is three females to two males. In surgical material, female preponderance is even greater, the ratio being eight to one (Lichtman, 1953).

In renal colic, by contrast with biliary colic, there were 6 females and 44 males. Five females and 42 males were under the age of 55. Winsbury-White (1954) gives an average age incidence of 40 years and a 5:2 male: female sex ratio.

\section{Discussion}

As mentioned in the opening paragraph, there are grounds for believing that a common misconception exists about the time/intensity pattern of pain in biliary and renal colic. It is probable that the word "colic" perpetuates this error, for previous descriptions have clearly made the point that the pain is usually continuous (Bockus, 1946 ; Adams and Stranahan, 1947). Ryle (1948) writes as follows: "The pains of biliary and renal colic, devastating in their ultimate throes, are nearly always continuous and crescendo, and in no true sense 'colicky': they start with a dull ache which becomes progressively more intolerable."

Analysis of the histories of 50 cases of biliary colic indicates that it is usual for the pain to rise to a level of severity which is maintained until the attack gradually subsides. This holds true whether the stone is too large to escape from the gall-bladder, or if there is subsequent jaundice, or even if the gall-bladder has been removed previously. There is no doubt that some of these attacks are associated with acute cholecystitis, but others are not, 
and there appears to be no clear distinction, either in the severity or in the duration of the attacks, which indicates the precise cause of the pain. Probably the pain may arise from one or more of several causes in a variety of sites in the biliary tract.

Judging from the data from 50 case histories of renal colic, the pattern of pain is often similar, but it is also common to have severe background pain, upon which bouts of even greater intensity are superimposed. In renal colic, infection can usually be excluded as a factor by examination of the urine. It is probable, therefore, that high pressure in the renal tract or smooth-muscle spasm, or a combination of these two, is responsible for the pain. That obstruction is present is strongly suggested by the fact that an I.V.P. during the attacks shows increased density of the kidney on the affected side with delay in the pyelogram. Evidence that a high pressure occurs proximal to the obstruction is provided by a recent experience. A man aged 49 developed typical right renal colic: an intravenous pyelogram was made about 45 minutes after the onset, when the pain was at its height. The first films showed a normal left pyelogram, an increase in the density of the right kidney, with a small stone at the lower end of the ureter, and later a delayed normal right pyelogram. A radiograph taken a few minutes later showed that the dye had burst out of the renal pelvis as a large cloud in the peripelvic tissues. Thirty-six hours after this there was right shoulder-tip pain, made worse by breathing, which persisted for 24 hours and then subsided without further incident. Retrograde pyelography a few days after the onset showed a normal renal pelvis, and the stone passed uneventfully.

\section{Summary}

A clinical study is made of the time/intensity pattern of pain in biliary and in renal colic. In biliary colic the pain rises to a plateau and does not usually show fluctuations in severity. Renal colic often has a similar pattern, but nearly half the patients noted some fluctuations in severity during an attack. Possible causes of these pains are discussed. Intermittent pain such as is implied by the word "colic" is rare.

Our thanks are due to Professor J. Bruce, Dr. W. I. Card, Mr. D. Band, and Mr. W. Selby Tulloch for permission to study their patients; and to Dr. M. B. Matthews and Dr. W. H. Price for criticism and advice in the preparation of the paper.

\section{REFERENCES}

Adams, R., and Stranahan, A. (1947). Surg. Gynec. Obstet., 85, 776.

Bockus, H. L. (1946). Gastroenterology. Saunders, Philadelphia and London.

Lichtman, S. S. (1953). Diseases of the Liver, Gallbladder, and Bile Duct. Kimpton London.

Ryle, J. A. (1948). Natural History of Disease, 2nd ed. Oxford Univ Press, London

Winsbury-White, H. P. (1954). Stone in the Urinary Tract, 2nd ed. Butterworth, London.

\title{
GALL-BLADDER DYSPEPSIA
}

\author{
BY
}

\section{W. H. PRICE, M.B., B.Sc., M.R.C.P.Ed Eastern General Hospital, Edinburgh}

The purpose of this inquiry was to examine the relationship between dyspepsia and gall-bladder disease. Patients who suffer from biliary colic or acute cholecystitis frequently deny a previous history of indigestion (Colp et al., 1935). Moreover, it is unfortunately the experience of many practitioners that patients are often not relieved of various types of chronic dyspepsia by cholecystectomy. Yet gaseous eructation, flatulence, heaviness, epigastric pains of varying intensity, and heartburn were described by Moynihan (1908) as "the inaugural symptoms of gallbladder disease." This view was shared by many wellknown medical men, including Mayo Robson, Bland-Sutton, and Mayo, and it is expressed in numerous textbooks. However, the literature contains conflicting opinions on the significance of so-called "gall-bladder dyspepsia." For instance, Bockus and Dozzi (1946) state: "We have become more and more convinced that dyspeptic symptoms occurring day after day are rarely due to uncomplicated cholelithiasis." Alvarez (1943) and Ivy et al. (1950) believe that such symptoms are due usually to functional disease of the gastro-intestinal tract, and are not connected with the gall-bladder.

The scheme of this investigation was to record the histories from a group of women who were then submitted to routine cholecystography. It was thought that in this way the relationship between various types of dyspepsia and the presence of gall-stones could be examined without bias.

\section{Choice of Subjects}

The inquiry was conducted within a general practice of about 3,000 patients in a densely populated urban area of whom approximately half were women. The survey was restricted to women aged 50-70. The reasons for this choice were: (1) it was desirable to include within the survey a reasonable proportion of subjects with gall-stones ; (2) gall-stones are known to be commoner in women than in men in the proportion of $3: 2$ (Bockus); and (3) the frequency of gall-stones rises with advancing years: over the age of 50 about one woman in five in this country has gall-stones (Bockus).

At the outset it was decided to exclude: (1) Eight women who, because of illness, were unable to attend the hospital for $x$-ray examination. Four of these were in mental hospitals; one was blind, deaf, and dumb; one had inoperable carcinoma ; and two were crippled by rheumatoid arthritis. (2) Five women upon whom cholecystectomy had been performed. These were excluded as it would not be possible to obtain an unbiased history, but they were later interviewed to determine as far as was possible the reason for operation and the effect it had had on pre-operative symptoms.

With these exceptions, all the women aged 50-70 were circularized by letter asking if they would co-operate in an investigation into indigestion. A second letter was sent three months later to those who did not reply. If there was still no response the address was visited. Information extracted from the N.H.S. card included: (1) Name, age, and address; (2) distance of the home from the surgery; (3) average annual surgery attendance for the previous three years ; and (4) previous complaint of dyspepsia.

Among the 204 women who were circularized 142 (70\%) were interviewed and $x$-rayed, while the other $62(30 \%)$ refused to co-operate. No difference was demonstrated between these two groups when compared by age, previous complaint of dyspepsia to the practitioner, and distance from the home to the surgery (Table I). Annual surgery 